\title{
AXIAL SHEAR MODULUS OF A FIBER-REINFORCED COMPOSITE WITH RANDOM FIBER CROSS-SECTIONS
}

\author{
S.K. BOSE \\ Department of Mathematics \\ Regional Engineering College \\ Durgapur-713209, India \\ and \\ L. DEBNATH \\ Mathematics and Physics Departments \\ East Carolina University \\ Greenville, North Carolina 27834, U.S.A. \\ (Received April 14, 1981)
}

ABSTRACT. A study is made of the effective axial shear modulus of a fiber reinforced material with random fiber cross-sections so that the micromechanics is governed by stochastic differential equations. A coarse-graining procedure is adopted to investigate the macroscopic behavior of the material. This analysis leads to the formula for the effective axial shear modulus,

$$
\mu *=\mu_{1} /\left\{1-2 c\left(\mu_{2}-\mu_{1}\right) /\left(\mu_{2}+\mu_{1}\right)\right\}
$$

where $\mu_{1}$ and $\mu_{2}$ are the shear modulus of the matrix and fibers respectively and $c$ is the concentration of the fibers less that 0.5 . For $c>0.5$, the fiber and matrix moduli are to be interchanged and $c$ is to be replaced by $1-c$. The results of this study are compared with those of the theory of fibre reinforced materials. Finally, a numerical example is presented with graphical representation.

KEY WORIS AND PHRASES. Axial shear modulus, Fiber-keinforced composites, Kandom distributions, Random cross-sections, Coarse-graining, Bounds on moduli.

\section{MATHEMATICS SUBJECT CLASSIFICATION CODE. $73 B 35$.}

\section{INTRODUCTION.}

From the view point of statistical continuum mechanics, a few theories have 
been developed for the effective viscosity and the effective thermal conductivity in random suspensions. Einstein's formula [1] is well known as the first theoretical result for the effective viscosity in dilute suspensions of shperical particles. In recent years, Beran [2] and Miller [3] have used perturbation methods combined with Green's functions to derive the expression for the effective thermal conductivity in suspensions. On the other hand, Hori [4], Hori and Yonezawa [5-8] have obtained the rigorous formula for the effective thermal conductivity in a random medium using diagrammatic expansions from a field-theoretical view point. In spite of this progress, no results are available for the effective viscosity in more concentrated suspensions.

In a very recent paper, Nagatani [9] has presented a general theory of the macroscopic motions of slow viscous flows in suspensions from the view point of statistical continuum mechanics. With the assumption that the characteristic length scale is much larger than the size of the suspended particles, he derived the coarse-grained equations from the governing equations using the formal perturbation methods and coarse-graining procedures. The macroscopic motions on much larger scale than the size of the suspended particles. It has been shown that this formula reduces to the Einstein formula for dilute suspensions. Nagatani's formula for the effective viscosity has a simple closed form and has been found to agree well with experimental results.

In the derivation of the formula, the suspension with its variable physical properties on a microscipic scale, is governed by stochastic differential equations. The macroscopic behavior of the system could be obtained by ensemble averaging the equations. However, this procedure is usually difficult and an alternative "coarsegraining" procedure is adopted. The coarse-graining means downgrading the spatial resolution to a much larger scale than that of a particle. This is achieved by looking at the particles as random perturbations and representing the field variables by Fourier series in the space co-ordinates. Modes with low wave numbers (lower than the reciprocal of the size of a particle) are retained while those with high wave numbers are repeatedly eliminated through a formal perturbation technique. This yields the expanded form of the effective viscosity, which when 
formally resumed gives the formula.

We adopt this procedure to determine the effective axial shear modulus of a random composite, with unidirectional fibers. This analysis leads to the formula for the effective axial shear modulus, $\mu^{*}=\mu_{1} /\left\{1-2 c\left(\mu_{2}-\mu_{1}\right) /\left(\mu_{2}+\mu_{1}\right)\right\}$ where $\mu_{1}$ and $\mu_{2}$ are the shear moduli of the matrix and fibers respectively and $c$ is the concentration of the fibers less than 0.5 . The fibers with their random cross-sections are looked upon as random perturbations over the matrix material, and as such the concentration $c$ of the fibers can not exceed 0.5 ( if $c$ exceeds 0.5 we can interchange the roles of the fibers and the matrix) - a range which is important from the practical point of view. For $c=0$, it reduces to the shear modulus of the matrix and for $c=0.5$ it yields the mean of the fiber and matrix moduli. This shows the consistency of the result, since the fiber geometry plays no role in the method, and is random. An important feature of the formula is its simple structure and can be of practical use. Even when the fibers are completely identical (which is difficult to ensure in practice) the formula would be approximately true.

The formula is compared with the bounds obtained by Hashin [10] and it is shown to exceed his lower bound. For higher values of the concentration, it, however, exceeds his upper bound also.

\section{BASIC STOCHASTIC EQUATIONS ON THE MICROSCOPIC SCALE.}

We consider a cylindrical specimen of the material with side surface parallel to the fibers. Let the specimen be in a state of shear in the axial direction. If we introduce co-ordinate axes $0 \mathrm{x}_{1}$ and $0 \mathrm{x}_{2}$ in a cross-section, the shear stress $\mathrm{T}_{j}$ on it is given by

$$
T_{j}=\mu \frac{\partial w}{\partial x_{j}}, \quad(j=1,2)
$$

where $w(\underset{d}{x}), \underset{\sim}{x}=\left(x_{1}, x_{2}\right)$ is the accompanying displacement in the axial direction and $\mu$ is the shear modulus of the material. If $\mu_{1}$ and $\mu_{2}$ are respectively the shear moduli of the materials of the matrix and the fibers, $\mu$ takes these values in the two phases. Looking upon the fibers as random perturbations over the matrix, 
we write

$$
\mu=\mu_{1}+\left(\mu_{2}-\mu_{1}\right) \xi(\underset{\sim}{x})
$$

where

$$
\begin{aligned}
\xi(x) & =0, \text { in the matrix } \\
& =1, \text { in the fibers. }
\end{aligned}
$$

The function $\xi(x)$ is a random variable with mean

$$
\langle\xi\rangle=\frac{1}{\mathrm{~S}} \int_{S} \xi(\underset{\mathrm{x}}{\mathrm{x}}) \mathrm{dx}=\mathrm{c}
$$

where $S$ is the area of the cross-section and $c$ is the 'concentration' of the fibers. In the statistical analysis of the problem, we shall also encounter the mean of $\left\{\xi(\underset{N}{x}\}^{n}\right.$. Noting from equation (2.3) that its value is that of $\xi(\underset{x}{)}$, we also have

$$
\left\langle\xi^{\mathrm{n}}\right\rangle=\mathrm{c}
$$

The stress equation of equilibrium of the material, with equation (2.1) becomes

$$
\frac{\partial}{\partial x_{j}}\left(\mu \frac{\partial w}{\partial x_{j}}\right)=0
$$

which apparant1y is a stochastic differential equation. On the microscale the displacement and stresses must be continuous across the interfaces of the two phases.

\section{COARSE-GRAINED EQUATIONS.}

In order to obtain the macroscopic behavior from the stochastic equations (2.1) - (2.6), we subject them to the coarse-graining procedure. I'his envisages downgrading the spatial resolution to a scale much larger than the cross-section of a fiber. To this end we represent the variables in equations (2.1) - (2.6) in Fourier series:

$$
\begin{aligned}
& T_{j}=s^{-\frac{1}{2}} \sum_{\underset{\sim}{k}} U_{j \underset{\sim}{k}} e^{i k \cdot x} \\
& w=s^{-\frac{1}{2}} \sum_{\sim} w_{\underline{\sim}} e^{i \underset{\sim}{k} \cdot x} \\
& \xi=s^{-\frac{1}{2}} \sum_{\underset{\sim}{k}} x_{\underline{\sim}} e^{i k \cdot x} \cdot
\end{aligned}
$$


The Fourier components in the above are given by

$$
\begin{aligned}
& U_{j \underset{\sim}{k}}=s^{-\frac{1}{2}} \int_{S} T_{j} e^{-i k i k} \cdot \underset{\sim}{x} d x \\
& \underset{\underline{k}}{w_{k}}=s^{-\frac{1}{2}} \int_{S} w e^{-i k} \cdot \underset{\sim}{d x} \underset{\sim}{d x} \\
& \underset{\sim}{x_{k}}=s^{-\frac{1}{2}} \int_{S} \xi e^{-i k} \cdot \underset{\sim}{d x}
\end{aligned}
$$

If we use equations $(2.2),(3.1),(3.2)$ and (3.3) in (2.1), we obtain

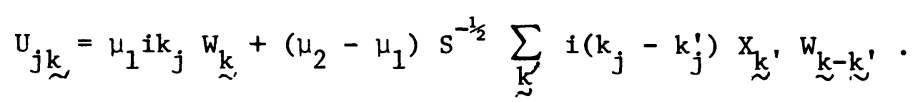

It is apparant that, in the summation on the right hand side, all the wave numbers $\mathrm{k}^{\prime}$ contribute to the component $U_{j k}$. If from this summation we separate out the term corresponding to the lowest wave number ${\underset{\sim}{k}}^{\prime}=0$, we get

$$
\begin{aligned}
\mathrm{U}_{j \underset{\sim}{k}}= & i k_{j}\left[\mu_{1}+\left(\mu_{2}-\mu_{1}\right)\langle\xi\rangle\right] w_{\sim} \\
& +\left(\mu_{2}-\mu_{1}\right) s^{-\frac{1}{2}} \sum_{k^{\prime} \neq 0} i\left(k_{j}-k_{j}^{\prime}\right) x_{k^{\prime}} \underbrace{}_{k_{k}-k^{\prime}},
\end{aligned}
$$

where we have used the relation

$$
\mathrm{x}_{0}=\mathrm{s}^{\frac{1}{2}}\langle\xi\rangle
$$

Adopting the same procedure for equation (2.6) with (2.2), (3.2) and (3.3), we get

$$
\mathrm{w}_{\mathrm{k}}=-\frac{\mu_{2}-\mu_{1}}{\mu_{1} \mathrm{k}^{2}} \mathrm{~s}^{-\frac{1}{2}} \sum_{\mathrm{k}^{\prime}} \mathrm{k}_{\mathrm{m}}\left(\mathrm{k}_{\mathrm{m}}-\mathrm{k}_{\mathrm{m}}^{\prime}\right) \mathrm{x}_{\mathrm{k}^{\prime}} \mathrm{w}_{\underline{\mathrm{k}}-\mathrm{k}^{\prime}} .
$$

In the coarse-graining procedure we repeatedly use equation (3.6) in the right hand side of (3.4) and remove the term corresponding to the high wave numbers $\underline{\mathrm{k}}^{\prime} \neq 0$ at each step. Physically this means downgrading the spatial resolution of the composite. As the wave numbers should occur with the diameter of a fiber in a natural way, the resolution would be on a scale much larger that the cross-section of a fiber. At the first step we have 


$$
\begin{aligned}
& \mathrm{U}_{j \underset{\sim}{k}}=i k_{j}\left[\mu_{1}+\left(\mu_{2}-\mu_{1}\right)\langle\xi\rangle\right] \mathrm{w}_{\underset{\sim}{k}} \\
& -\frac{\left(\mu_{2}-\mu_{1}\right)^{2}}{\mu_{1}} s^{-1} w_{\underset{k}{k}} \cdot \sum_{\mathfrak{k}^{\prime} \neq 0} i k_{m} \frac{\left(k_{j}-k_{j}^{\prime}\right)\left(k_{m}-k_{m}^{\prime}\right)}{\left(\underset{\sim}{k}-\underset{\sim}{\left.k^{\prime}\right)^{2}}\right.} x_{\underbrace{\prime}} x_{-\underset{N}{k}}
\end{aligned}
$$

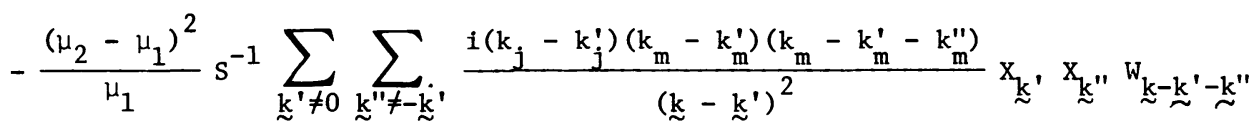

If we assume that the composite is statistically isotropic, the second order tensor $\left(k_{j}-k_{j}^{\prime}\right)\left(k_{m}-k_{m}^{\prime}\right)$ is approximately an isotropic tensor of order two with

$$
\frac{\left(k_{j}-k_{j}^{\prime}\right)\left(k_{m}-k_{m}^{\prime}\right)}{\left(\underset{\sim}{k}-k^{\prime}\right)^{2}} \approx \frac{1}{2} \delta_{j m}
$$

The second term on the right hand side of (3.7) thus becomes

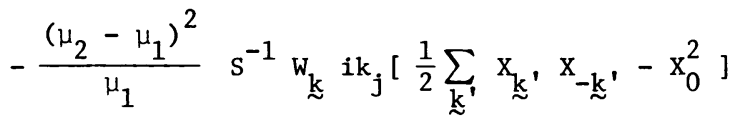

$$
\begin{aligned}
& =-\frac{\left(\mu_{2}-\mu_{1}\right)^{2}}{\mu_{1}} i k_{j}{\underset{\sim}{k}}_{\underset{k}{ }}\left[\frac{1}{2}\left\langle\xi^{2}\right\rangle-\langle\xi\rangle^{2}\right] \text {, }
\end{aligned}
$$

where we have used the relation (3.5) and the equation (3.3) to obtain

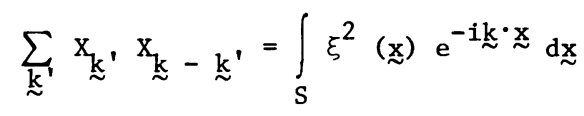

This leads to the result

$$
\sum_{k^{\prime}} x_{k^{\prime}} x_{-k^{\prime}}=s\left\langle\xi^{2}\right\rangle \text { for } \underset{\sim}{k}=0
$$

At the next step of the coarse-graining procedure, we again replace $\mathrm{w}_{\mathbf{k}}-\mathrm{k}^{\prime}-\mathrm{k}^{\prime \prime}$ by the approximate expression from (3.6) and remove the term corresponding to $\mathrm{k}^{\prime} \neq 0$. The contribution of this term is

$$
\frac{\left(\mu_{2}-\mu_{1}\right)^{3}}{\mu_{1}^{2}} i k_{j} w_{\sim}\left[\frac{1}{4}\langle\xi\rangle-\langle\xi\rangle\left\langle\xi^{2}\right\rangle+\langle\xi\rangle^{3}\right] .
$$

Continuing this process, we get from (3.7)

$$
\mathrm{U}_{j \underset{\sim}{\mathrm{k}}}=\mu^{*} i \mathrm{k}_{\mathrm{j}} \mathrm{W}_{\underline{\mathrm{k}}},
$$


where

$$
\begin{aligned}
\mu^{*}=\mu_{1}[1 & +\frac{\mu_{2}-\mu_{1}}{\mu_{1}}\langle\xi\rangle-\frac{\left(\mu_{2}-\mu_{1}\right)^{2}}{\mu_{1}^{2}}\left\{\frac{1}{2}\left\langle\xi^{2}\right\rangle-\langle\xi\rangle^{2}\right\} \\
& \left.+\frac{\left(\mu_{2}-\mu_{1}\right)^{3}}{\mu_{1}^{3}}\left\{\frac{1}{4}\left\langle\xi^{3}\right\rangle-\langle\xi\rangle\left\langle\xi^{2}\right\rangle+\langle\xi\rangle^{3}\right\}-\ldots\right] .
\end{aligned}
$$

With equations (3.1) and (3.2), the result (3.10) yields

$$
T_{j}=\mu^{*} \frac{\partial w}{\partial x_{j}} \text {. }
$$

The above being the mean stress-strain relation, $\mu^{*}$ is the effective axial shear modulus of the composite.

4. EFFECTIVE AXIAL SHEAR MODULUS.

If we use equations (2.4) and (2.5) in (3.11), we get the expanded form of $\mu$ * in terms of $c$. If the infinite series is summed formally, we get the closed form

$$
\frac{\mu *}{\mu_{1}}=\frac{1}{1-\left\{\frac{2\left(\mu_{2}-\mu_{1}\right)}{\mu_{2}+\mu_{1}} c\right\}}
$$

The expression (4.1) gives the correct value $\mu_{1}$ when $c=0$. When $\mu_{2}>\mu_{1}$, it increases with c. However, when c approaches $\left(\mu_{2}+\mu_{1}^{j}\right) /\left[2\left(\mu_{2}-\mu_{1}\right)\right]>\frac{1}{2}$, it becomes infinitely large. However, we note that we have looked upon the fibers as random perturbations over the matrix material and as such $c$ should not exceed 0.5 . For $c=0.5$ we get $\mu^{*}=\left(\mu_{1}+\mu_{2}\right) / 2$, which is a consistent result, as the fiber geometry is random. If $c$ exceeds 0.5 we should interchange the roles of the fibers and the matrix and we have

$$
\frac{\mu^{*}}{\mu_{2}}=\frac{1}{1-\left\{\frac{2\left(\mu_{1}-\mu_{2}\right)}{\mu_{1}+\mu_{2}}(1-c)\right\}} \text {. }
$$

This case is, however, not very important from a practical point of view.

Hashin [10] has given lower and upper bounds for $\mu^{*}$. If these are denoted by $\mu_{L}^{*}$ and $\mu_{U}^{*}$, then in our notations

$$
\frac{\mu_{L}^{*}}{\mu_{1}}=\frac{\mu_{2}+\mu_{1}+c\left(\mu_{2}-\mu_{1}\right)}{\mu_{2}+\mu_{1}-c\left(\mu_{2}-\mu_{1}\right)} \text {, }
$$




$$
\frac{\mu_{U}^{*}}{\mu_{1}}=\frac{\mu_{2}}{\mu_{1}}\left[\frac{\mu_{2}+\mu_{1}-(1-c)\left(\mu_{2}-\mu_{1}\right)}{\mu_{2}+\mu_{1}+(1-c)\left(\mu_{2}-\mu_{1}\right)}\right] \text {. }
$$

It easily follows from (4.1) and (4.2) that $\mu^{*}>\mu_{L}^{*}$. Also the rate of increase of $\mu^{*}$ is higher than that of $\mu_{L}^{*}$. For higher concentrations the deviation from the lower bound will then be pronounced. This behavior is generally in agreement with experiments. In relation to the upper bound however, $\mu^{*}>\mu_{\mathrm{U}}^{*}$ for $c>\left(\mu_{2}-\mu_{1}\right) /\left(2 \mu_{2}\right)<\frac{1}{2}$.

5. NUMERICAL EXAMPLE.

We consider Boron fibers in Aluminum matrix. For such a composite $\mu_{2} / \mu_{1}=$ $25 / 3.87$. We can easily compute the normalized shear moduli given by equations (4.1), (4.2) and (4.3) for different values of the concentration c. The results are graphically presented in Fig. 1:

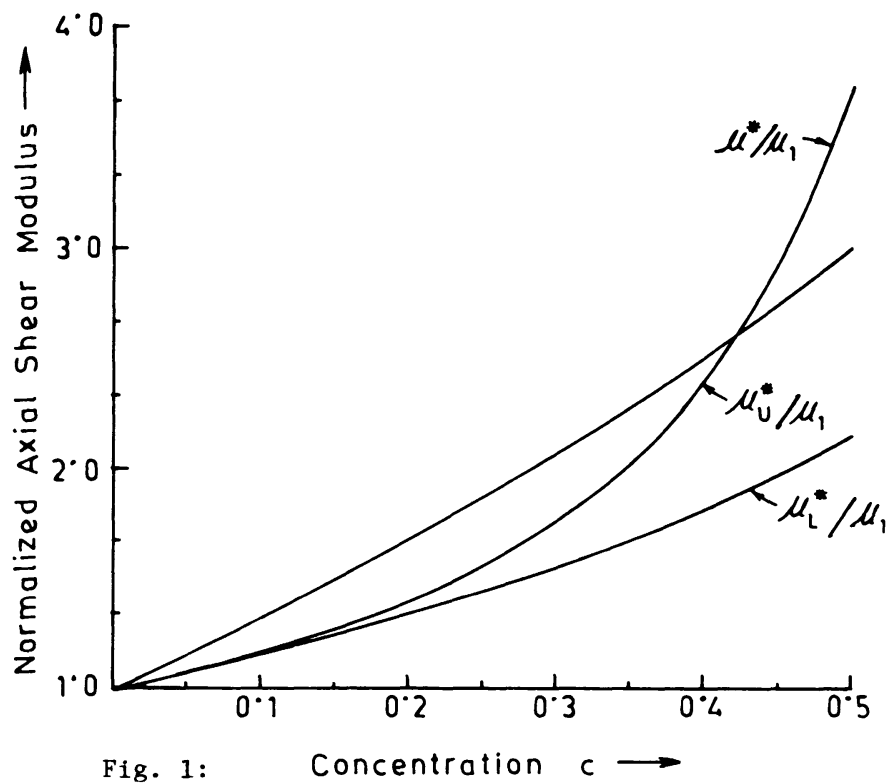

The figure indicates that $\mu^{\star}$ is closer to the lower bound for values of $c$ up to 0.35 ; thereafter, it is closer to the upper bound.

ACKNOWLEDGEMENT. The second author wishes to express his grateful thanks to East Carolina University for partial support of this work. 


\section{REFERENCES}

1. EINSTEIN, A. Zur Theorie des Brownschen Bewegung, Ann. Phys. 19 (1906) 371-381.

2. BERAN, M.J. Statistical Continuum Theories, Interscience Publisher (1968).

3. MILLER, M.N. Bounds for Effective Electrica1, Thermal, and Magnetic Properties of Heterogeneous Materials, J. Math. Phys. 10 (1969) 1988-2004.

4. HORI, M. Statistical Theory of Effective Electrical, Therma1, and Magnetic Properties of Random Heterogeneous Materials, VII. Comparison of Different Approaches, J. Math. Phys. 18 (1977) 487-501.

5. HORI, M. and YONEZAWA, F. Statistical Theory of Effective Electrical, Thermal, and Magnetic Properties of Random Heterogeneous Materia1s, III. Perturbation treatment of the effective permittivity in completely random heterogeneous materials, J. Math. Phys. 15 (1974) 2177-2185.

6. HORI, M. and YONEZAWA, F. Theoretical Approaches to in homogeneous transport in disordered media, J. Phys. C10 (1977) 229-248.

7. HORI, M. and YONEZAWA, F. Statistical Theory of Effective Electrical, Therma1, and Magnetic Properties of Random Heterogeneous Materials, V. One- and two-dimensional systems, J. Math. Phys. 16 (1975) 365-377.

8. HORI, M. and YONEZAWA, F. Statistical Theory of Effective Electrical, Thermal, and Magnetic Properties of Random Heterogeneous Materia1s, IV. Effective Medium Theory and Cumulant Expansion Method, J. Math. Phys. 16 (1975) 352-358.

9. NAGATANI, T. Statistical Theory of Effective Viscosity in a Random Suspension, J. Phys. Soc. Jap. 47 (1979) 320-326.

10. HASHIN, Z. Theory of Fiber Reinforced Materials, NASA CR-1974 (1972). 


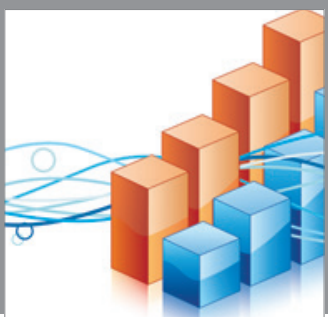

Advances in

Operations Research

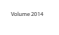

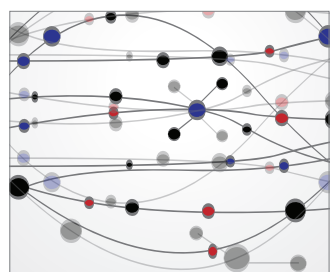

\section{The Scientific} World Journal
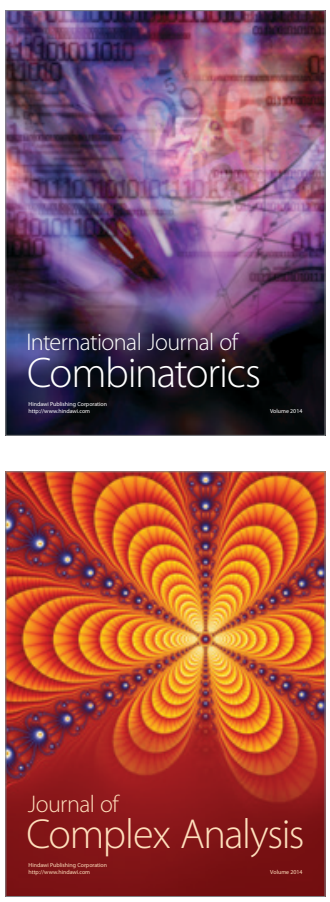

International Journal of

Mathematics and

Mathematical

Sciences
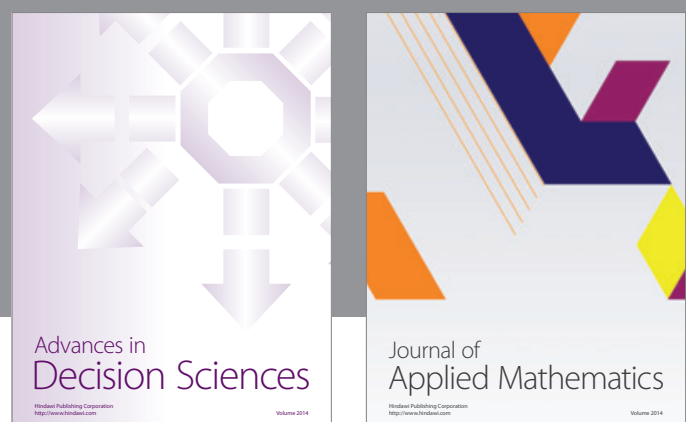

Journal of

Applied Mathematics
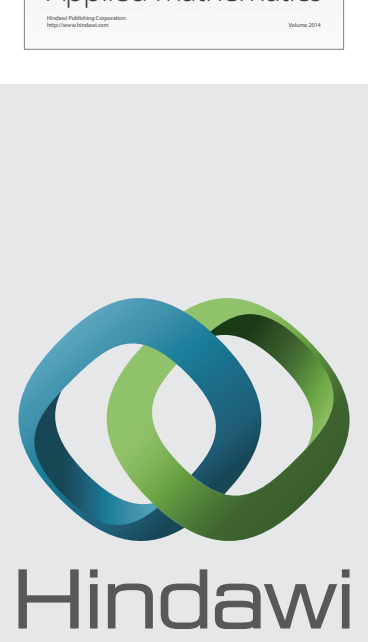

Submit your manuscripts at http://www.hindawi.com
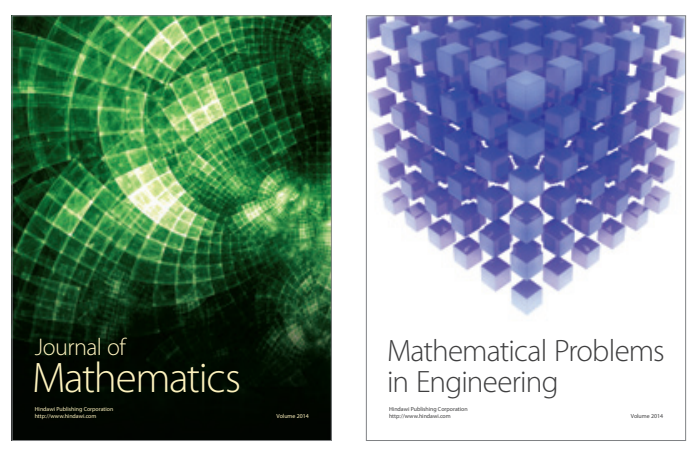

Mathematical Problems in Engineering
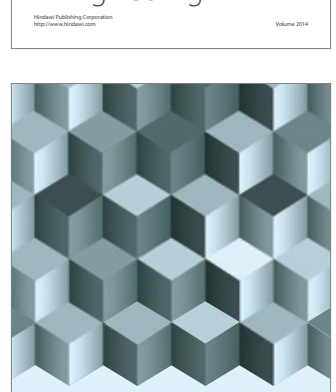

Journal of

Function Spaces
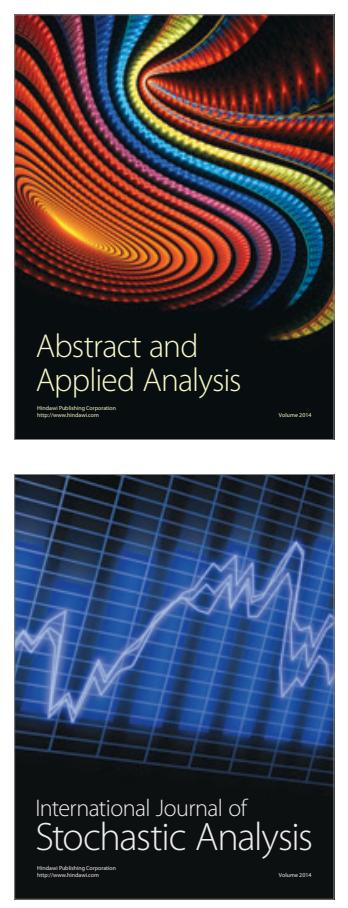

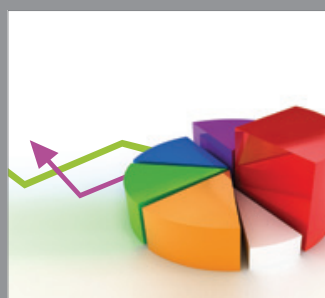

ournal of

Probability and Statistics

Promensencen
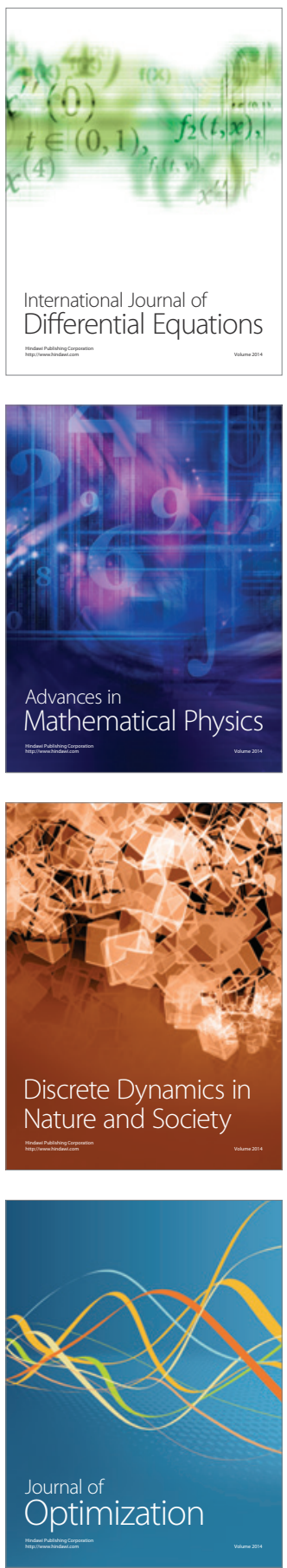